\title{
Inflaton two-point correlation in the presence of a cosmic string
}

\author{
Chien-Yao Tseng and Mark B. Wise \\ California Institute of Technology, Pasadena, California 91125, USA
}

(Received 8 September 2009; published 13 November 2009)

\begin{abstract}
Precise measurements of the microwave background anisotropy have confirmed the inflationary picture of approximately scale invariant, Gaussian primordial adiabatic density perturbations. However, there are some anomalies that suggest a small violation of rotational and/or translational invariance in the mechanism that generates the primordial density fluctuations. Motivated by this we study the two-point correlation of a massless scalar (the inflaton) when the stress tensor contains the energy density from an infinitely long straight cosmic string in addition to a cosmological constant.
\end{abstract}

DOI: 10.1103/PhysRevD.80.103512

PACS numbers: $98.80 . \mathrm{Cq}$

\section{INTRODUCTION}

The inflationary cosmology is the standard paradigm for explaining the horizon problem [1,2]. In its simplest form inflation predicts an almost scale invariant spectrum of approximately Gaussian density perturbations [3,4]. Rotational and translational invariance dictate that the two-point correlation of the Fourier transform of the primordial density perturbations $\delta(\mathbf{k})$ has the form,

$$
\langle\delta(\mathbf{k}) \delta(\mathbf{q})\rangle=P(k)(2 \pi)^{3} \delta(\mathbf{k}+\mathbf{q}),
$$

where $k=|\mathbf{k}|$ and $P$ is called the power spectrum. In Eq. (1) the fact that $P$ only depends on the magnitude of the wave vector $\mathbf{k}$ is a consequence of rotational invariance and the delta function of $\mathbf{k}+\mathbf{q}$ arises from translational invariance. Let $\chi(\mathbf{k})$ be the Fourier transform of a massless scalar field with canonical normalization. Its two-point correlation in de Sitter space is

$$
\langle\chi(\mathbf{k}) \chi(\mathbf{q})\rangle=P_{\chi}(k)(2 \pi)^{3} \delta(\mathbf{k}+\mathbf{q}),
$$

where $H$ is the Hubble constant during inflation and

$$
P_{\chi}(k)=\frac{H^{2}}{2 k^{3}} \text {. }
$$

In the inflationary cosmology the almost scale invariant density perturbations that are probed by the microwave background anisotropy and the large scale structure of our observed Universe have a power spectrum that differs from $P_{\chi}(k)$ normalization factor that has weak $k$ dependence ${ }^{1}$ and a transfer function that arises from the growth of fluctuations at late times after they reenter the horizon [5-13].

Inflation occurs at an early time when the energy density of the universe is large compared to energy scales that can be probed by laboratory experiments. It is possible that there are paradigm shifts in our understanding of the laws of nature, as radical as the shift from classical physics to quantum physics, that are needed to understand physics at the energy scale associated with the inflationary era.

\footnotetext{
${ }^{1}$ We will treat this factor as a constant and denote it by $\kappa^{2}$.
}

Motivated by the lack of direct probes of physics at the inflationary scale Ackerman et al. wrote down the general form that Eq. (1) would take [14] if rotational invariance was broken by a small amount during the inflationary era (but not today) by a preferred direction and computed its impact on the microwave background anisotropy (see also [15-21]). They also wrote down a simple field theory model that realizes this form for the density perturbations where the preferred direction is associated with spontaneous breaking of rotational invariance by the expectation value of a vector field. This model serves as a nice pedagogical example, however, it cannot be realistic because of instabilities [22]. Evidence in the WMAP data for the violation of rotational evidence was found in Refs. [2325]. Another anomaly in the data on the anisotropy of the microwave background data is the "hemisphere effect" $[26,27]$. This cannot be explained by the model of Ackerman et al. Erickeck et al. proposed an explanation based on the presence of a very long wavelength (superhorizon) perturbation [28]. This long wavelength mode picks out a preferred wave number and can give rise to a hemisphere effect. It violates translational invariance, and there are very strong constraints from the observed large scale structure of the Universe on this [29-31]. The generation of large-scale temperature fluctuations in the microwave background temperature by superhorizon perturbations is known as the Grishchuk-Zel'dovich effect [32].

Carroll, et al. proposed explicit forms for violations of translational invariance [33], in the energy density perturbation two-point correlation, motivated by: the symmetries that are left unbroken, the desire to have a prediction for the two-point correlation of multipole moments of the microwave background anisotropy $\left\langle a_{l m} a_{l^{\prime} m^{\prime}}^{*}\right\rangle$ that is nonzero for at most a few $l$ 's that are different from $l^{\prime}$, and the desire to introduce at most a few new parameters. To get a feeling for what can happen in general consider a case where there is a special point $\mathbf{x}_{0}$ during inflation. Its presence violates translational invariance; however, translational invariance is restored if in addition to translating the spatial coordinates we also translate $\mathbf{x}_{0}$. So in coordi- 
nate space $\langle\delta(\mathbf{x}) \delta(\mathbf{y})\rangle$ must be a function of $\mathbf{x}, \mathbf{y}$ and $\mathbf{x}_{0}$ that is invariant under translations $\mathbf{x} \rightarrow \mathbf{x}+\mathbf{a}, \mathbf{y} \rightarrow \mathbf{y}+\mathbf{a}$, $\mathbf{x}_{0} \rightarrow \mathbf{x}_{0}+\mathbf{a}$ and rotations $\mathbf{x} \rightarrow R \mathbf{x}, \mathbf{y} \rightarrow R \mathbf{y}, \mathbf{x}_{0} \rightarrow R \mathbf{x}_{0}$. Furthermore, it must be symmetric under interchange of $\mathbf{x}$ and $\mathbf{y}$. Ref. [33] assumed $\langle\delta(\mathbf{x}) \delta(\mathbf{y})\rangle$ only depends on the two variables, $\left(\mathbf{x}-\mathbf{x}_{0}\right)^{2}+\left(\mathbf{y}-\mathbf{x}_{0}\right)^{2}$ and $|\mathbf{x}-\mathbf{y}|$, and expanded in the dependence the first of these. However, in the general case of a special point $\mathbf{x}_{0}$ Eq. (1) becomes

$$
\langle\delta(\mathbf{k}) \delta(\mathbf{q})\rangle=e^{i(\mathbf{k}+\mathbf{q}) \cdot \mathbf{x}_{0}} P(k, q, \mathbf{k} \cdot \mathbf{q}),
$$

where $P$ is symmetric under interchange of $\mathbf{k}$ and $\mathbf{q}$. Without further simplifying assumptions about the form of $P$ and the value of $\mathbf{x}_{0}$ this will result in a very complicated matrix ${ }^{2}\left\langle a_{l m} a_{l^{\prime} m^{\prime}}^{*}\right\rangle$.

In this paper we explore the form of the two-point correlation $\langle\delta(\mathbf{k}) \delta(\mathbf{q})\rangle$ if translational invariance is broken by the presence of cosmic string that passes through our horizon volume during inflation. We will assume that the string becomes unstable and disappears near the end of inflation and approximate the string as infinitely long and having infinitesimal thickness. In that case rotational invariance about the string axis and translational invariance along the string direction are left unbroken. Aligning the preferred direction with the $z$ axis these symmetries imply that the two-point correlation of energy density correlations takes the form

$$
\begin{aligned}
\langle\delta(\mathbf{k}) \delta(\mathbf{q})\rangle= & (2 \pi) \delta\left(k_{z}+q_{z}\right) e^{i\left(\mathbf{k}_{\perp}+\mathbf{q}_{\perp}\right) \cdot \mathbf{x}_{0}} \\
& \times P\left(k_{\perp}, q_{\perp}, k_{z}, \mathbf{k}_{\perp} \cdot \mathbf{q}_{\perp}\right),
\end{aligned}
$$

with $P$ symmetric under the interchange of $\mathbf{k}_{\perp}$ and $\mathbf{q}_{\perp}$. Here, we have decomposed the wave vectors along the $z$ axis and the two-dimensional subspace perpendicular to that is denoted by a subscript $\perp$. $\mathbf{x}_{0}$ is a point on the string. If the preferred direction is along an arbitrary direction $\hat{\mathbf{n}}=R \hat{\mathbf{z}}$, where $R$ is a rotation that leaves the point $\mathbf{x}_{0}$ fixed, then on the right-hand side of Eq. (5) the wave vectors are replaced by the rotated ones; $\mathbf{k} \rightarrow R \mathbf{k}$ and $\mathbf{q} \rightarrow R \mathbf{q}$. The goal of this paper is to derive an explicit expression for the function $P\left(k_{\perp}, q_{\perp}, k_{z}, \mathbf{k}_{\perp} \cdot \mathbf{q}_{\perp}\right)$.

Using cylindrical spatial coordinates the metric for the inflationary space-time with an infinitely long infinitesimally thin straight string directed along $z$ direction and passing through the origin is [34]

$$
d s^{2}=-d t^{2}+a(t)^{2}\left[d \rho^{2}+\rho^{2}(1-4 G \mu)^{2} d \theta^{2}+d z^{2}\right],
$$

where $a(t)=e^{H t}$ is just the ordinary inflationary scale factor and $\mu$ is the tension along the string. We compute the Fourier transform of the two-point correlation of $\chi$ in this space-time. This is a simplified model for inflation where $\chi$ plays the role of the inflaton and $\delta(\mathbf{k}) \propto \chi(\mathbf{k})$. We focus on the cosmic string case because of the simplicity of

\footnotetext{
${ }^{2} l, m$ label the rows and $l^{\prime}, m^{\prime}$ the columns.
}

the metric and not because of a strong physical motivation. Unless there is " just enough inflation" it is very unlikely that there would be a cosmic string in our horizon volume during inflation. If there was just enough inflation [35-37] there could be other sources of violations of translational and rotational invariance [38-43]. However, the cosmic string case does provides a simple physical model where the form of the violation of translational and rotational invariance can be explicitly calculated, and it depends on only the parameter $G \mu$ and four other parameters that specify the location and alignment of the string. Real cosmic strings have a thickness of order $1 / \sqrt{\mu}$ and so for it to be treated as thin we need $H^{2}<<\mu$, which implies that the dimensionless quantity $\epsilon=G \mu$ is much greater than, $G H^{2}$.

It is also possible to violate translational invariance by a point defect that existed during the inflationary era. In the conclusions we briefly discuss how the cosmic string case differs from the case of a black hole located in our horizon volume during the inflationary era [44].

\section{THE TWO-POINT CORRELATION FUNCTION OF A MASSLESS SCALAR}

The metric for an inflationary space-time with an infinitely long string along the $z$ direction and through the origin is taken of the form [34]

$$
d s^{2}=-d t^{2}+a(t)^{2}\left[d \rho^{2}+\rho^{2}(1-4 G \mu)^{2} d \theta^{2}+d z^{2}\right],
$$

where $a(t)=e^{H t}$ is just the ordinary inflationary scale factor. We let $\alpha=1-4 G \mu$. In these coordinates the Lagrangian density for a massless scalar field is

$$
\begin{aligned}
\mathcal{L}_{\chi}= & -\frac{\sqrt{-g}}{2} g^{\mu \nu} \partial_{\mu} \chi \partial_{\nu} \chi \\
= & \frac{a(t)^{3}}{2} \rho \alpha\left(\frac{\partial \chi}{\partial t}\right)^{2}-\frac{a(t)}{2} \rho \alpha\left(\frac{\partial \chi}{\partial z}\right)^{2} \\
& -\frac{a(t)}{2} \rho \alpha\left(\frac{\partial \chi}{\partial \rho}\right)^{2}-\frac{a(t)}{2 \rho \alpha}\left(\frac{\partial \chi}{\partial \theta}\right)^{2} .
\end{aligned}
$$

The Hamiltonian ${ }^{3}$ is,

$$
\begin{aligned}
H= & \int d^{3} x(\pi \dot{\chi}-\mathcal{L}) \\
= & \int \rho d \rho d \theta d z \frac{\alpha}{2}\left[a(t)^{3}\left(\frac{\partial \chi}{\partial t}\right)^{2}+a(t)\left(\frac{\partial \chi}{\partial z}\right)^{2}\right. \\
& \left.+a(t)\left(\frac{\partial \chi}{\partial \rho}\right)^{2}+\frac{a(t)}{\rho^{2} \alpha^{2}}\left(\frac{\partial \chi}{\partial \theta}\right)^{2}\right] .
\end{aligned}
$$

It is convenient to introduce the conformal time

\footnotetext{
${ }^{3}$ The same symbol is used for the Hamiltonian and Hubble constant during inflation; however, the meaning of the symbol should be clear from the context.
} 


$$
\tau=-\frac{1}{H} e^{-H t}
$$

and as $t$ goes from $-\infty$ to $\infty$ the conformal time $\tau$ goes from $-\infty$ to 0 . Since the metric only differs from de Sitter space by the presence of a conical singularity at $\rho=0$ the (equal time) two-point correlation of $\chi$ can easily shown to be

$$
\begin{aligned}
\left\langle\chi(\rho, \theta, z, \tau) \chi\left(\rho^{\prime}, \theta^{\prime}, z^{\prime}, \tau\right)\right\rangle= & \int_{0}^{\infty} \frac{d k_{\perp}}{2 \pi} k_{\perp} \int_{-\infty}^{\infty} \frac{d k_{z}}{2 \pi} e^{i k_{z}\left(z-z^{\prime}\right)} \\
& \times \sum_{m=-\infty}^{\infty} \frac{e^{i m\left(\theta-\theta^{\prime}\right)}}{2 \pi} J_{|m / \alpha|}\left(k_{\perp} \rho\right) \\
& \times J_{|m / \alpha|}\left(k_{\perp} \rho^{\prime}\right) \frac{\left|\chi_{k}(\tau)\right|^{2}}{\alpha} .
\end{aligned}
$$

Here, $\chi_{k}(\tau)$ are the usual mode functions in de Sitter space

$$
\chi_{k}(\tau)=\frac{H}{\sqrt{2 k}} e^{-i k \tau}\left(\tau-\frac{i}{k}\right) .
$$

We are interested in the late time, $k \tau \rightarrow 0$ behavior. Using the explicit form of $\chi_{k}(\tau)$ above,

$$
\begin{aligned}
\left\langle\chi(\rho, \theta, z, 0) \chi\left(\rho^{\prime}, \theta^{\prime}, z^{\prime}, 0\right)\right\rangle & \\
= & \frac{H^{2}}{2 \alpha} \int_{0}^{\infty} \frac{d k_{\perp}}{2 \pi} k_{\perp} \int_{-\infty}^{\infty} \frac{d k_{z}}{2 \pi} e^{i k_{z}\left(z-z^{\prime}\right)} \\
& \times \sum_{m=-\infty}^{\infty} \frac{e^{i m\left(\theta-\theta^{\prime}\right)}}{2 \pi} \frac{J_{|m / \alpha|}\left(k_{\perp} \rho\right) J_{|m / \alpha|}\left(k_{\perp} \rho^{\prime}\right)}{\left(k_{\perp}^{2}+k_{z}^{2}\right)^{3 / 2}} .
\end{aligned}
$$

The observed Universe is consistent with the standard predictions of the inflationary cosmology. Therefore, the violation of translational invariance due to the string is a small perturbation, and is parametrized by the small quantity $\epsilon=4 G \mu$. There are two approaches to calculate the Fourier transform of the two-point correlation of $\chi$. One is to expand the Bessel functions in Eq. (13) about $\epsilon=0$ and then change to Cartesian coordinates. Another approach, which is the one we take, is to abandon the exact result in Eq. (13) and just do quantum mechanical perturbation theory about the unperturbed, $\epsilon=0$ background, i.e., de Sitter space.

In the standard inflationary cosmology with one field, the inflaton, perturbations in the gauge invariant quantity, that reduces to the density perturbations for modes with wavelengths well within the horizon, are calculated from the two-point function of a massless scalar field. Precisely how this field is related to the gravitational and scalar degrees of freedom depends on the choice of gauge. One can work in a gauge where the scalar field has no fluctuations and then the massless field lives in the gravitational degrees of freedom. (See [45] for a calculation in this gauge.). For a more conventional approach where fluctuations in the inflaton field itself are computed, see, for example, [46]. We assume a similar calculation holds in the case we are discussing so we approach the problem by computing the fluctuations in a massless scalar field $\chi$ and take, $\delta=\kappa \chi$. We need to compute the two-point correlation function $\langle\chi(\mathbf{x}, t) \chi(\mathbf{y}, t)\rangle$. Treating $\epsilon$ as a small perturbation and using the "in-in" formalism, to first order of $\epsilon$, (see Ref. [47]).

$$
\begin{aligned}
\langle\chi(\mathbf{x}, t) \chi(\mathbf{y}, t)\rangle \simeq & \left\langle\chi_{I}(\mathbf{x}, t) \chi_{I}(\mathbf{y}, t)\right\rangle \\
& +i \int_{-\infty}^{t} d t^{\prime} e^{-\epsilon^{\prime}\left|t^{\prime}\right|} \\
& \times\left\langle\left[H_{I}\left(t^{\prime}\right), \chi_{I}(\mathbf{x}, t) \chi_{I}(\mathbf{y}, t)\right]\right\rangle,
\end{aligned}
$$

where $\epsilon^{\prime}$ is an infinitesimal parameter that cuts off the early time part of the integration. In this case the interactionpicture Hamiltonian $H_{I}(t)$ is given by

$$
\begin{aligned}
H_{I}= & \int \rho d \rho d \theta d z\left(-\frac{\epsilon}{2}\right)\left[a^{3}\left(\frac{\partial \chi_{I}}{\partial t}\right)^{2}+a\left(\frac{\partial \chi_{I}}{\partial z}\right)^{2}\right. \\
& \left.+a\left(\frac{\partial \chi_{I}}{\partial \rho}\right)^{2}-\frac{a}{\rho^{2}}\left(\frac{\partial \chi_{I}}{\partial \theta}\right)^{2}\right] \\
= & \int \rho d \rho d \theta d z\left(-\frac{\epsilon}{2}\right)\left[a^{3}\left(\frac{\partial \chi_{I}}{\partial t}\right)^{2}+a\left(\frac{\partial \chi_{I}}{\partial z}\right)^{2}\right. \\
& \left.+a\left(\frac{\partial \chi_{I}}{\partial \rho}\right)^{2}+\frac{a}{\rho^{2}}\left(\frac{\partial \chi_{I}}{\partial \theta}\right)^{2}-\frac{2 a}{\rho^{2}}\left(\frac{\partial \chi_{I}}{\partial \theta}\right)^{2}\right] \\
= & -\epsilon H_{0}+\epsilon \int \rho d \rho d \theta d z \frac{a}{\rho^{2}}\left(\frac{\partial \chi_{I}}{\partial \theta}\right)^{2},
\end{aligned}
$$

where the interaction-picture field $\chi_{I}$ has its time evolution governed by the unperturbed Hamiltonian

$$
\begin{aligned}
H_{0}= & \int \rho d \rho d \theta d z \frac{1}{2}\left[a^{3}\left(\frac{\partial \chi_{I}}{\partial t}\right)^{2}+a\left(\frac{\partial \chi_{I}}{\partial z}\right)^{2}\right. \\
& \left.+a\left(\frac{\partial \chi_{I}}{\partial \rho}\right)^{2}+\frac{a}{\rho^{2}}\left(\frac{\partial \chi_{I}}{\partial \theta}\right)^{2}\right] .
\end{aligned}
$$

Because we are interested in the effects that violate rotational and/or translational invariance in, $\Delta\langle\chi(\mathbf{x}, t) \chi(\mathbf{y}, t)\rangle=$ $\langle\chi(\mathbf{x}, t) \chi(\mathbf{y}, t)\rangle-\left\langle\chi_{I}(\mathbf{x}, t) \chi_{I}(\mathbf{y}, t)\right\rangle$, we will drop the term proportional to $H_{0}$ in the interaction Hamiltonian leaving us with

$$
H_{I}=\epsilon \int \rho d \rho d \theta d z \frac{a}{\rho^{2}}\left(\frac{\partial \chi_{I}}{\partial \theta}\right)^{2},
$$

to first order in $\epsilon$. The free field obeys the unperturbed equation of motion

$$
\frac{d^{2} \chi_{I}}{d t^{2}}+3 H \frac{d \chi_{I}}{d t}-\frac{1}{a(t)^{2}} \frac{d^{2} \chi_{I}}{d \mathbf{x}^{2}}=0 .
$$

Upon quantization, $\chi_{I}$ becomes a quantum operator 


$$
\begin{aligned}
\chi_{I}(\mathbf{x}, \tau)= & \int \frac{d^{3} k}{(2 \pi)^{3}} e^{i \mathbf{k} \cdot \mathbf{x}}\left[\chi_{k}(\tau) \beta(\mathbf{k})+\chi_{k}^{*}(\tau) \beta^{\dagger}(-\mathbf{k})\right] \\
= & \int \frac{d^{3} k}{(2 \pi)^{3}} e^{i k_{z} z} e^{i k_{\perp} \rho \cos \left(\theta-\theta_{k}\right)}\left[\chi_{k}(\tau) \beta(\mathbf{k})\right. \\
& \left.+\chi_{k}^{*}(\tau) \beta^{\dagger}(-\mathbf{k})\right],
\end{aligned}
$$

where $\chi_{k}(\tau)$ is given by Eq. (12). Note that we have converted to the conformal time $\tau=-e^{-H t} / H$ and used cylindrical coordinates for $\mathbf{k}$ and $\mathbf{x}$ in the exponential. $\beta(\mathbf{k})$ annihilates the vacuum state and satisfies the usual commutation relations $\left[\beta(\mathbf{k}), \beta^{\dagger}(\mathbf{q})\right]=(2 \pi)^{3} \delta(\mathbf{k}-\mathbf{q})$. Combining these definitions that interaction Hamiltonian can be written in terms of creation and annihilation operators as

$$
\begin{aligned}
H_{I}\left(\tau^{\prime}\right)= & \epsilon\left(\frac{1}{H \tau^{\prime}}\right) \int \frac{d^{3} k}{(2 \pi)^{3}} \int \frac{d^{3} q}{(2 \pi)^{3}} \\
& \times \int d^{3} x^{\prime} e^{i \mathbf{k} \cdot \mathbf{x}^{\prime}+i \mathbf{q} \cdot \mathbf{x}^{\prime}} \frac{\left(y^{\prime} k_{x}-x^{\prime} k_{y}\right)\left(y^{\prime} q_{x}-x^{\prime} q_{y}\right)}{x^{\prime 2}+y^{\prime 2}} \\
& \times\left[\chi_{k}\left(\tau^{\prime}\right) \beta(\mathbf{k})+\chi_{k}^{*}\left(\tau^{\prime}\right) \beta^{\dagger}(-\mathbf{k})\right]\left[\chi_{q}\left(\tau^{\prime}\right) \beta(\mathbf{q})\right. \\
& \left.+\chi_{q}^{*}\left(\tau^{\prime}\right) \beta^{\dagger}(-\mathbf{q})\right] .
\end{aligned}
$$

Next, we use the above results to compute the needed commutator $\quad\left\langle\left[H_{I}\left(\tau^{\prime}\right), \chi_{I}(\mathbf{x}, \tau) \chi_{I}(\mathbf{y}, \tau)\right]\right\rangle=\left\langle\left[H_{I}\left(\tau^{\prime}\right)\right.\right.$, $\left.\left.\chi_{I}(\mathbf{x}, \tau)\right] \chi_{I}(\mathbf{y}, \tau)\right\rangle+\left\langle\chi_{I}(\mathbf{x}, \tau)\left[H_{I}\left(\tau^{\prime}\right), \chi_{I}(\mathbf{y}, \tau)\right]\right\rangle$. This gives

$$
\begin{aligned}
& \left\langle\left[H_{I}\left(\tau^{\prime}\right), \chi_{I}(\mathbf{x}, \tau) \chi_{I}(\mathbf{y}, \tau)\right]\right\rangle \\
& =\frac{H^{3} \epsilon}{2 \tau^{\prime}} \int \frac{d^{3} k}{(2 \pi)^{3}} \int \frac{d^{3} q}{(2 \pi)^{3}} \int d^{3} x^{\prime} e^{i \mathbf{k} \cdot\left(\mathbf{x}^{\prime}-\mathbf{x}\right)+i \mathbf{q} \cdot\left(\mathbf{x}^{\prime}-\mathbf{y}\right)} \\
& \quad \times \frac{\left(y^{\prime} k_{x}-x^{\prime} k_{y}\right)\left(y^{\prime} q_{x}-x^{\prime} q_{y}\right)}{\left(x^{\prime 2}+y^{\prime 2}\right)} \frac{1}{k q}\left[e^{-i(k+q)\left(\tau^{\prime}-\tau\right)}\right. \\
& \left.\quad \times\left(\tau^{\prime}-\frac{i}{k}\right)\left(\tau^{\prime}-\frac{i}{q}\right)\left(\tau+\frac{i}{k}\right)\left(\tau+\frac{i}{q}\right)-\text { H.c. }\right] .
\end{aligned}
$$

Converting the integration over $t^{\prime}$ in Eq. (14) to the integration over the conformal time $\tau^{\prime}\left(d t^{\prime}=-\frac{d \tau^{\prime}}{H \tau^{\prime}}\right)$, using Eq. (21), and noting that the cutoff involving $\epsilon^{\prime}$ removes the influence at the very early time, we integrate over $\tau^{\prime}$ to get

$$
\begin{aligned}
\Delta\langle\chi(\mathbf{x}, \tau) \chi(\mathbf{y}, \tau)\rangle= & -H^{2} \epsilon \int \frac{d^{3} k}{(2 \pi)^{3}} \int \frac{d^{3} q}{(2 \pi)^{3}} \\
& \times \int d^{3} x^{\prime} e^{i \mathbf{k} \cdot\left(\mathbf{x}^{\prime}-\mathbf{x}\right)+i \mathbf{q} \cdot\left(\mathbf{x}^{\prime}-\mathbf{y}\right)} \\
& \times \frac{\left(y^{\prime} k_{x}-x^{\prime} k_{y}\right)\left(y^{\prime} q_{x}-x^{\prime} q_{y}\right)}{x^{\prime 2}+y^{\prime 2}} \\
& \times\left[\frac{k q+q^{2}+k^{2}+k^{2} q^{2} \tau^{2}}{k^{3} q^{3}(k+q)}\right] .
\end{aligned}
$$

Using

$$
\begin{aligned}
\frac{\left(y^{\prime} k_{x}-x^{\prime} k_{y}\right)\left(y^{\prime} q_{x}-x^{\prime} q_{y}\right)}{x^{\prime 2}+y^{\prime 2}}= & \mathbf{k}_{\perp} \cdot \mathbf{q}_{\perp} \\
& -\frac{\left(\mathbf{x}_{\perp}^{\prime} \cdot \mathbf{k}_{\perp}\right)\left(\mathbf{x}_{\perp}^{\prime} \cdot \mathbf{q}_{\perp}\right)}{x_{\perp}^{\prime}{ }^{2}},
\end{aligned}
$$

gives

$$
\begin{aligned}
& \Delta\langle\chi(\mathbf{x}, \tau) \chi(\mathbf{y}, \tau)\rangle=-H^{2} \epsilon \int \frac{d^{3} k}{(2 \pi)^{3}} \int \frac{d^{3} q}{(2 \pi)^{3}} \\
& \times e^{-i \mathbf{k} \cdot \mathbf{x}-i \mathbf{q} \cdot \mathbf{y}} 2 \pi \delta\left(k_{z}+q_{z}\right) \\
& \times\left[\frac{k q+q^{2}+k^{2}+k^{2} q^{2} \tau^{2}}{k^{3} q^{3}(k+q)}\right] \\
& \times\left[\int d ^ { 2 } x _ { \perp } ^ { \prime } e ^ { i ( \mathbf { k } _ { \perp } + \mathbf { q } _ { \perp } ) \cdot \mathbf { x } _ { \perp } ^ { \prime } } \left(\mathbf{k}_{\perp} \cdot \mathbf{q}_{\perp}\right.\right. \\
& \left.\left.-\frac{\left(\mathbf{x}_{\perp}^{\prime} \cdot \mathbf{k}_{\perp}\right)\left(\mathbf{x}_{\perp}^{\prime} \cdot \mathbf{q}_{\perp}\right)}{x_{\perp}^{\prime}{ }^{2}}\right)\right],
\end{aligned}
$$

where $x_{\perp}^{\prime}=\left|\mathbf{x}_{\perp}^{\prime}\right|$. It remains to perform the integration over $x^{\prime}$. We find that

$$
\begin{aligned}
\int d^{2} x_{\perp}^{\prime} e^{i \mathbf{p}_{\perp} \cdot \mathbf{x}_{\perp}^{\prime}} \frac{x_{\perp i}^{\prime} x_{\perp j}^{\prime}}{x_{\perp}^{\prime}{ }^{2}}= & (2 \pi)^{2} \delta\left(\mathbf{p}_{\perp}\right) \frac{\delta_{i j}}{2} \\
& +\frac{4 \pi}{p_{\perp}{ }^{2}}\left(\frac{\delta_{i j}}{2}-\frac{p_{\perp i} p_{\perp j}}{p_{\perp}{ }^{2}}\right)
\end{aligned}
$$

and so

$$
\begin{aligned}
\Delta\langle\chi(\mathbf{x}, \tau) \chi(\mathbf{y}, \tau)\rangle= & -H^{2} \epsilon \int \frac{d^{3} k}{(2 \pi)^{3}} \\
& \times \int \frac{d^{3} q}{(2 \pi)^{3}} e^{-i \mathbf{k} \cdot \mathbf{x}-i \mathbf{q} \cdot \mathbf{y}} 2 \pi \delta\left(k_{z}+q_{z}\right) \\
& \times \frac{k q+q^{2}+k^{2}+k^{2} q^{2} \tau^{2}}{k^{3} q^{3}(k+q)}\left[\frac{\mathbf{k}_{\perp} \cdot \mathbf{q}_{\perp}}{2}\right. \\
& \times(2 \pi)^{2} \delta\left(\mathbf{k}_{\perp}+\mathbf{q}_{\perp}\right)-\frac{4 \pi}{\left(\mathbf{k}_{\perp}+\mathbf{q}_{\perp}\right)^{2}} \\
& \times\left(\frac{\mathbf{k}_{\perp} \cdot \mathbf{q}_{\perp}}{2}\right. \\
& \left.\left.-\frac{\mathbf{k}_{\perp} \cdot\left(\mathbf{k}_{\perp}+\mathbf{q}_{\perp}\right) \mathbf{q}_{\perp} \cdot\left(\mathbf{k}_{\perp}+\mathbf{q}_{\perp}\right)}{\left(\mathbf{k}_{\perp}+\mathbf{q}_{\perp}\right)^{2}}\right)\right] .
\end{aligned}
$$

The second term in the large square brackets of Eq. (26) appears naively to give rise to a logarithmic divergence in the integrations over $q$ and $k$ near $\mathbf{p}_{\perp}=\mathbf{k}_{\perp}+\mathbf{q}_{\perp}=0$. However, after doing the angular integration over the direction of $\mathbf{p}_{\perp}$ this potentially divergent term vanishes.

Writing the density perturbations as $\delta=\kappa \chi$ we arrive at the following expression for the part of $P\left(k_{\perp}, q_{\perp}, k_{z}, \mathbf{k}_{\perp}\right.$. $\left.\mathbf{q}_{\perp}\right)$ in Eq. (5) that violates rotational and/or translational invariance: 


$$
\begin{aligned}
\Delta P\left(k_{\perp}, q_{\perp},\right. & \left.k_{z}, \mathbf{k}_{\perp} \cdot \mathbf{q}_{\perp}\right) \\
= & -\kappa^{2} H^{2} \epsilon\left(\frac{k q+q^{2}+k^{2}}{k^{3} q^{3}(k+q)}\right)\left[\frac{\mathbf{k}_{\perp} \cdot \mathbf{q}_{\perp}}{2}(2 \pi)^{2}\right. \\
& \times \delta\left(\mathbf{k}_{\perp}+\mathbf{q}_{\perp}\right)-\frac{4 \pi}{\left(\mathbf{k}_{\perp}+\mathbf{q}_{\perp}\right)^{2}}\left(\frac{\mathbf{k}_{\perp} \cdot \mathbf{q}_{\perp}}{2}\right. \\
& \left.\left.-\frac{\mathbf{k}_{\perp} \cdot\left(\mathbf{k}_{\perp}+\mathbf{q}_{\perp}\right) \mathbf{q}_{\perp} \cdot\left(\mathbf{k}_{\perp}+\mathbf{q}_{\perp}\right)}{\left(\mathbf{k}_{\perp}+\mathbf{q}_{\perp}\right)^{2}}\right)\right] .
\end{aligned}
$$

In Eq. (27) $k=\sqrt{k_{z}^{2}+k_{\perp}^{2}}$ and $q=\sqrt{k_{z}^{2}+q_{\perp}^{2}}$. Equation (27) is the main result of this paper. Notice that the dependence on the wave-vectors is scale invariant, which is mainly due to the assumption of massless inflaton. However, since the actual inflaton potential $V(\chi)$ steepens towards the end of inflation, there will be a scale-dependent spectral tilt on cosmologically observable scales. Furthermore, the scale invariance is also broken by the dependence on $\mathbf{x}_{0}$ that arises when one considers a string that does not pass through the origin. The first term in the large square brackets of Eq. (27) violates rotational invariance but not translational invariance. It is consistent with the form proposed by Ackerman et al. [14]. The second term in the large square brackets of Eq. (27) violates translational invariance.

Recall that in the model we have adopted the unperturbed density perturbations that have a power spectrum $P_{0}(k)=\kappa^{2} H^{2} /\left(2 k^{3}\right)$ and so $\epsilon$ characterizes the overall strength of the violations of rotational and translational invariance. For $\left(\mathbf{k}_{\perp}+\mathbf{q}_{\perp}\right) \cdot \mathbf{x}_{0}>>1$ the exponential dependence on this quantity in Eq. (5) oscillates rapidly, and this suppresses the impact of $\Delta P$ on observable quantities, which depend on integrals of $\langle\delta(\mathbf{k}) \delta(\mathbf{q})\rangle$ over the components of $\mathbf{q}$ and $\mathbf{k}$. Over some range of $k$ these oscillations may be observable. For a discussion of density perturbations that are modulated by an oscillating term see [48].

\section{CONCLUSIONS}

We have computed (with some simplifying assumptions) the impact that an infinitely long and infinitesimally thin straight string, that exists during inflation and passes through our horizon volume, has on the perturbations of the energy density of the Universe. We have assumed that the string disappears toward the end of the inflationary era. It may be possible to remove some of these assumptions or provide dynamics that realizes them. However, even without that, Eq. (27) provides a simple and physically motivated functional form (after modifying it so the string can have an arbitrary location and orientation) for the part of the density perturbations two-point correlation function that violates translational and rotational invariance. It can be compared with data on the large-scale structure of the Universe and the anisotropy of the microwave background radiation.

Computations analogous to those performed in this paper can be done for a point defect (located at the origin $x=$ 0 ) in de Sitter space using the metric $[49,50]$

$$
\begin{aligned}
d s^{2}= & -\frac{\left(1-\frac{r_{0}}{a(t) x}\right)^{2}}{\left(1+\frac{r_{0}}{a(t) x}\right)^{2}} d t^{2} \\
& +a(t)^{2}\left(1+\frac{r_{0}}{a(t) x}\right)^{4}\left(d x^{2}+x^{2} d \Omega_{2}^{2}\right),
\end{aligned}
$$

where $a(t)=e^{H t}$. In this case, perturbing about de Sitter space, yields the following interaction Hamiltonian for a massless scalar field $\chi$,

$$
H_{I}=4 r_{0} \int d^{3} x\left(\frac{1}{x}\right) a(t)^{2}\left(\frac{d \chi_{I}}{d t}\right)^{2}=4 r_{0} \int d^{3} x\left(\frac{1}{x}\right)\left(\frac{d \chi_{I}}{d \tau}\right)^{2} .
$$

For the point mass case the perturbative calculation of $\langle\chi(\mathbf{x}, t) \chi(\mathbf{y}, t)\rangle$ using Eq. (14) has greater sensitivity to earlier times $t^{\prime}$. For example, the factor of $e^{-\epsilon^{\prime}\left|t^{\prime}\right|}$ does not regulate the $t^{\prime}$ integration. An exponential regulator in conformal time would work but then the Fourier transform of the part of this two-point function that violates translation invariance vanishes as $q \tau$ and $k \tau$ go to zero. This case was considered in Ref. [44].

\section{ACKNOWLEDGMENTS}

We thank S. Carroll for discussions particularly during the early stages of this work. We are grateful for the Department of Energy for partial support under Grant No. DE-FG03-92ER40701.
[1] A. H. Guth, Phys. Rev. D 23, 347 (1981).

[2] A. D. Linde, Phys. Lett. 108B, 389 (1982); A. Albrecht and P. J. Steinhardt, Phys. Rev. Lett. 48, 1220 (1982).

[3] V.F. Mukhanov and G. V. Chibisov, Pis'ma Zh. Eksp. Teor. Fiz. 33, 549 (1981) [JETP Lett. 33, 532 (1981)]; A. A. Starobinsky, Phys. Lett. 117B, 175 (1982); A. H. Guth and S. Y. Pi, Phys. Rev. Lett. 49, 1110 (1982); S. W.
Hawking, Phys. Lett. 115B, 295 (1982).

[4] For a review of inflation see e.g. S. Dodelson, Modern Cosmology (Academic Press, San Diego, 2003); D. Langlois, arXiv:hep-th/0405053.

[5] G.F. Smoot et al., Astrophys. J. 396, L1 (1992); C. L. Bennett et al., Astrophys. J. 464, L1 (1996)

[6] J. E. Ruhl et al., Astrophys. J. 599, 786 (2003); S. Masi 
et al., arXiv:astro-ph/0507509; W.C. Jones et al., Astrophys. J. 647, 823 (2006);

[7] M. C. Runyan et al., Astrophys. J. Suppl. Ser. 149, 265 (2003); C.1. Kuo et al. (ACBAR Collaboration), Astrophys. J. 600, 32 (2004).

[8] T. J. Pearson et al., Astrophys. J. 591, 556 (2003); A. C. S. Readhead et al., Astrophys. J. 609, 498 (2004).

[9] P. F. Scott et al., Mon. Not. R. Astron. Soc. 341, 1076 (2003); K. Grainge et al., Mon. Not. R. Astron. Soc. 341, L23 (2003); C. Dickinson et al., extended Mon. Not. R. Astron. Soc. 353, 732 (2004).

[10] A. Benoit et al. (Archeops Collaboration), Astron. Astrophys. 399, L19 (2003); arXiv:astro-ph/0210305; M. Tristram et al., Astron. Astrophys. 436, 785 (2005).

[11] N. W. Halverson et al., Astrophys. J. 568, 38 (2002).

[12] A. T. Lee et al., Astrophys. J. 561, L1 (2001).

[13] D. N. Spergel et al., Astrophys. J. Suppl. Ser. 170, 377 (2007); G. Hinshaw et al., Astrophys. J. Suppl. Ser. 170, 288 (2007).

[14] L. Ackerman, S. M. Carroll, and M. B. Wise, Phys. Rev. D 75, 083502 (2007).

[15] A. E. Gumrukcuoglu et al., arXiv:astro-ph/0608405.

[16] C. Armendariz-Picon, J. Cosmol. Astropart. Phys. 09 (2007) 014.

[17] T. S. Pereira et al., J. Cosmol. Astropart. Phys. 09 (2007) 006.

[18] A. E. Gumrukcuoglu et al., J. Cosmol. Astropart. Phys. 11 (2007) 005.

[19] S. Yokoyama and J. Soda, J. Cosmol. Astropart. Phys. 08 (2008) 005.

[20] S. Kanno, M. Kimura, J. Soda, and S. Yokoyama, J. Cosmol. Astropart. Phys. 08 (2008) 034.

[21] M. A. Watanabe, S. Kanno, and J. Soda, Phys. Rev. Lett. 102, 191302 (2009); V. A. Kostelecky and S. Samuel, Phys. Rev. D 40, 1886 (1989).

[22] B. Himmetoglu et al., Phys. Rev. Lett. 102,111301 (2009).

[23] A. R. Pullen and M. Kamionkowski, Phys. Rev. D 76, 103529 (2007).

[24] N. E. Groeneboom and H. K. Eriksen, Astrophys. J. 690, 1807 (2009).
[25] C. Armendariz-Picon and L. Pekowsky, Phys. Rev. Lett. 102, 031301 (2009).

[26] H. K. Eriksen et al., Astrophys. J. 605, 14 (2004); 609, 1198(E) (2004).

[27] H. K. Eriksen et al., Astrophys. J. 660, L81 (2007).

[28] A. L. Erickcek et al., Phys. Rev. D 78, 123520 (2008).

[29] S. Ho, C. M. Hirata, N. Padmanabhan, U. Seljak, and N. Bahcall, Phys. Rev. D 78, 043519 (2008).

[30] C. M. Hirata, S. Ho, N. Padmanabhan, U. Seljak, and N. Bahcall, Phys. Rev. D 78, 043520 (2008).

[31] C. M. Hirata, J. Cosmol. Astropart. Phys. 09 (2009) 011.

[32] L. P. Grishchuk and Ya. B. Zeldovich, Sov. Astron. 22, 125 (1978); M. S. Turner, Phys. Rev. D 44, 3737 (1991).

[33] S. M. Carroll et al., arXiv:astro-ph/0811.1086.

[34] A. H. Abbassi, A. M. Abbassi, H. Razmi, Phys. Rev. D 67, 103504 (2003).

[35] N. Deruelle, and D. S. Goldwirth, Phys. Rev. D 51, 1563 (1995).

[36] D. S. Goldwirth, Phys. Rev. D 43, 3204 (1991).

[37] D. S. Goldwirth and T. Piran, Phys. Rep. 214, 223 (1992).

[38] A. Aguirre, M. C. Johnson, and A. Shomer, Phys. Rev. D 76, 063509 (2007).

[39] S. Chang et al., J. Cosmol. Astropart. Phys. 04 (2009) 025.

[40] A. Aguirre and M. C. Johnson, Phys. Rev. D 77, 123536 (2008).

[41] A. Aguirre, M. C. Johnson, and M. Tysanner, Phys. Rev. D 79, 123514 (2009).

[42] S. Chang et al., J. Cosmol. Astropart. Phys. 04 (2008) 034.

[43] B. Freivogel et al., J. Cosmol. Astropart. Phys. 08 (2009) 036.

[44] H.-T. Cho et al., arXiv:astro-ph/0905.2041v1.

[45] J. Maldacena, J. High Energy Phys. 05 (2003) 013.

[46] S. Dodelson, Modern Cosmology (Academic Press, San Diego, 2003).

[47] S. Weinberg, Phys. Rev. D 72, 043514 (2005).

[48] J. Martin and C. Ringeval, Phys. Rev. D 69, 083515 (2004); J. Cosmol. Astropart. Phys. 01 (2005) 007.

[49] G. C. McVittie, Mon. Not. R. Astron. Soc. 93, 325 (1933).

[50] C. J. Gao, Classical Quantum Gravity 21, 4805 (2004). 